\title{
Investigation of Free Space Optical Detection Module Operating at the Wavelength Range of 8-12 $\mu \mathrm{m}$
}

\author{
M. Gutowska ${ }^{a}$, M. Nowakowski $^{a}$, Z. Bielecki $^{a}$, J. MikolajczyK ${ }^{a}$, D. Szabra ${ }^{a}$,

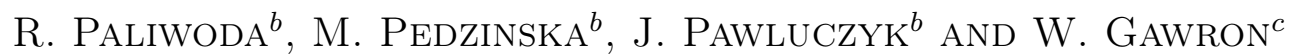 \\ ${ }^{a}$ Institute of Optoelectronics, Military University of Technology, S. Kaliskiego 2, 00-908 Warsaw, Poland \\ ${ }^{b}$ Vigo System S.A, Poznańska 129/133, 05-850 Ożarów Mazowiecki, Poland \\ ${ }^{c}$ Institute of Applied Physics, Military University of Technology, S. Kaliskiego 2, 00-908 Warsaw, Poland \\ The paper presents the construction and test results of a long-term radiation detector module for free \\ space optical communication operated at the wavelength range of 8-12 $\mu \mathrm{m}$. In the module a Polish detector \\ manufactured in the company Vigo System S.A. was applied. High sensitivity of the detection module was \\ achieved through a multi-layered heterostructure $\mathrm{HgCdTe}$ with immersion lens, which is optimized for the radiation \\ detection at the wavelength of $10 \mu \mathrm{m}$. Detector noises were reduced as a result of detector cooling by means of \\ four-stage thermoelectric cooler. The developed detection module is dedicated to design a next-generation optical \\ data link. The link will be characterized by a greater range in difficult weather conditions in relation to the \\ currently offered links operating at the shorter wavelengths.
}

PACS numbers: 07.07.Df, 85.60.Bt

\section{Introduction}

Development of broadband optical telecommunications in free space (free space optics - FSO) has shown that the technology operating at the wavelength range about $10 \mu \mathrm{m}$ is very promising [1]. A significant advantage of the technology in comparison with currently available optical communications systems is lesser scattering of radiation by aerosols, clouds and dust. This is particularly important in urban areas and in conditions of high smoke e.g. on the battlefield $[2,3]$. Additionally, the fact that this wavelength range of radiation is also considered safe for the eyes makes this technology very interesting.

Until recently, the lack of suitable radiation sources and detectors has constituted the main problem area. The existing devices were expensive and not user-friendly. It appears that the problem of radiation sources may be solved by the development of quantum cascade lasers [4].

Optical data link usually consists of two identical sets of receiver-transmitter. Typically, a suitable source of radiation, control system for this source and optics are the most important elements of the transmitter. The receiver is built from a detection module and an optics.

\section{Detection module for free-space optical communication}

The main requirements for a detection module are determined by level of power $\left(P_{0}\right)$ registered by the photoreceiver. The power can be calculated from the formula

$$
P_{0}=P_{n} \frac{D_{0}^{2}}{\left(R \theta_{n}\right)^{2}} \tau T,
$$

where $P_{n}$ - power of radiation source, $\tau$ - atmospheric transmission, $T$ - factor determined by transmissions of transmitter and photoreceiver optics, $D_{0}$ - surface area of the receiver optics, $R$ - transmitter-receiver distance, $\theta_{n}$ - beam divergence.

It could be noticed that the parameters of data link are generally determined by the parameters of the photoreceiver, for example noise level or size of photoreceiver optics. An important parameter of communication links is bit error rate (BER). Ordinarily, the parameter is determined by

$$
\mathrm{BER}=\frac{1}{2} \operatorname{erfc}\left(\frac{\sqrt{\mathrm{SNR}}}{2 \sqrt{2}}\right),
$$

where: erfc - complementary error function, SNR signal to noise rate of the photoreceiver.

Basing on the formulae (1) and (2), the main requirements for long-wave detectors for free-space optical communication can be defined:

- High sensitivity. High sensitivity provides to achieve low error ratio using low laser beam power and small-aperture optics. In practice, fundamental limits are required.

- High operation speed. The present optical links are characterized by sub-nanosecond response time.

- Other requirements: user-friendly, reliable, inexpensive and well-fitted to the beam spot size in the focal plane of an optical system. 


\section{Photodetectors operating at the wavelength range of $8-12 \mu \mathrm{m}$}

The photoreceiver detection module PVI-4TE-10.6 type with multilayer $\mathrm{Hg}_{1-x} \mathrm{Cd}_{x}$ Te heterostructure constructed at Polish Vigo Ltd. Co. was applied. The photodiode structure is composed of 6-20 layers obtained from low-temperature metal organic chemical vapour deposition epitaxy. This technology has been discussed in detail in papers [5-7]. This detector responsivity is optimized for the wavelengths of about $10 \mu \mathrm{m}$ at the operation temperature of about $230 \mathrm{~K}$. The temperature stabilization is assured by four-stages TE-cooler (Fig. 1). High per-

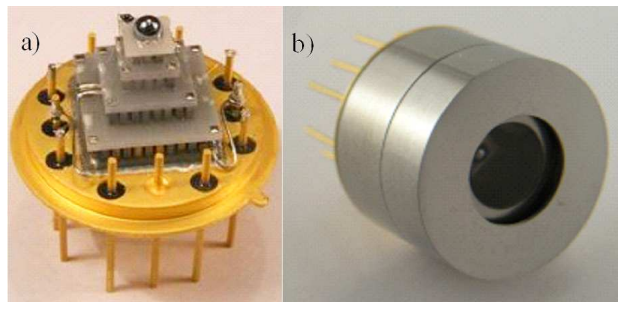

Fig. 1. Optically immersed detectors mounted on cold finger of the four-stage Peltier coolers (a), detector housing (b).

formance and stability were achieved by using band gap engineered $(\mathrm{HgCdZn}) \mathrm{Te}$ structures of optimized doping and improved surface processing [8].

The device architecture has been optimized with the use of computer-aided simulation. The thicknesses of subsequent layers, band gap profiles, types and levels of donor and acceptor doping have been obtained from calculations. The results are as follows:

- optimal relation between absorption of exact wavelength radiation and the thermal generation rate of carriers in the absorber area,

- minimized thermal generation and recombination of carriers in contact and transient areas, and on the surface of the heterostructure,

- elimination of short-wave radiation noise by adequate choice of $N^{+}$layer composition,

- good and fast collection of optically generated carriers,

- minimized parasitic impedances at the mesa structure base, wide band gap contact areas and at the contact between the heterostructure and the metallization,

- minimized $R C$ time constant.

The contact metallization additionally functions as a mirror reflecting low-absorption long-wave radiation back to the absorber. Buffer, absorber and contact layer thicknesses are chosen to create a resonant cavity along with the contact metallization. The cavity is not particularly perfect but offers an increase in device quantum efficiency in the long-wave range $[9,10]$.

The photodiode heterostructure is monolithically integrated with an immersion lens which functions as an effective optical concentrator (Fig. 1). In the lens, the optical area is increased $n^{2}$ times the physical area, where $n$ is the refraction index of the lens material. The applied technology provides a radical decrease in the thermal generation-recombination of carriers and the noise power [9, 11]. Additionally, an electric capacity is reduced by decreasing the absorber area. This results in a radical drop in the $R C$ time constant. However, application of the lenses has negative influence. The technology increases in manufacturing costs and, in the case of hyperhemispheric lenses, limits a field of view and decreases in radiation saturation threshold. Summarizing, immersed detector construction provides an increase in detectivity and operating speed as compared to conventional, non-immersed one.

\section{Detector investigations}

Detector investigations were made using a specially prepared laboratory setup. The main experimental task was to measure a current-voltage, dark resistancevoltage, and spectral sensitivity characteristics. The obtained characteristic of dynamic resistance versus bias voltage is presented in Fig. 2.

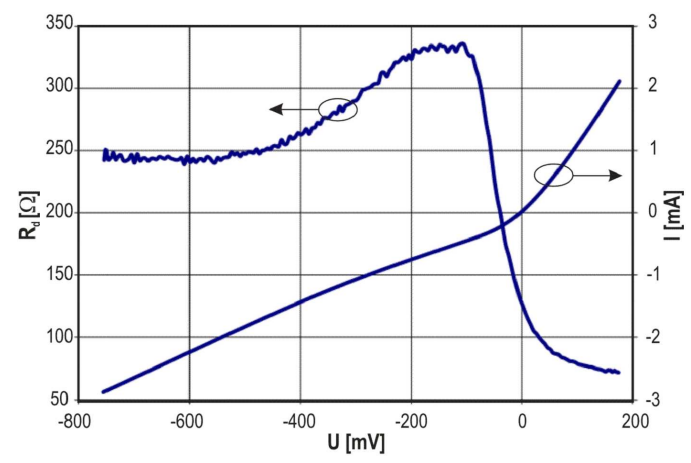

Fig. 2. Dynamic resistance $R_{\mathrm{d}}$ vs. bias voltage (optical area of $\left.0.3 \times 0.3 \mathrm{~mm}^{2}\right)$.

The photodiode detectivity is specified on the basis of current sensitivity and $R_{0}$ :

$$
D^{*}=\frac{R_{i} A^{1 / 2}}{\left(4 k T R_{0}^{-1}\right)^{1 / 2}},
$$

where $k$ - Boltzmann's constant, $T$ - operating temperature, $R_{i}$ - current sensitivity, $R_{0}$ - resistance (no bias).

Figure 3 presents a normalized detectivity of the photodiode versus wavelengths. The photodiodes are characterized by long-wave photosensitivity of about $13 \mu \mathrm{m}$.

The time constant measurement of the photoreceiver was made using an oscilloscope and a high repetition 


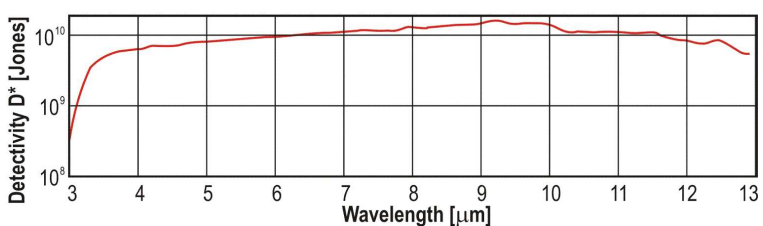

Fig. 3. Spectral characteristics of normalized detectivity $D^{*}$.

radiation source. During measurement 20 ps radiation pulses with a wavelength $\lambda=10 \mu \mathrm{m}$ were applied. The output signal of the detector was gained by a preamp with a $300 \mathrm{MHz}$ signal band and registered by an oscilloscope with $8 \mathrm{GHz}$ bandwidth. The measured time constant of the photodetector was approximately $3.9 \mathrm{~ns}$.

The parameters of the photodetector are listed in Table.

Parameters of the $\mathrm{Hg}_{1-x} \mathrm{Cd}_{x}$ Te photodetector.

TABLE

\begin{tabular}{l|c|c}
\hline \hline \multicolumn{1}{c|}{ Parameter } & Units & Value \\
\hline ambient temperature & $\mathrm{K}$ & 293 \\
thermistor resistance & $\mathrm{k} \Omega$ & 472 \\
detector resistance & $\Omega$ & 122 \\
optical area & $\mathrm{mm}^{2}$ & 0.09 \\
reverse bias voltage & $\mathrm{mV}$ & 0 \\
current responsivity $\pm 20 \%(10 \mu \mathrm{m})$ & $\mathrm{A} / \mathrm{W}$ & 4.1 \\
current noise density & $\mathrm{pA} / \mathrm{Hz}^{1 / 2}$ & 9.2 \\
detectivity $\pm 20 \%(10 \mu \mathrm{m})$ & $\mathrm{J}$ & $1.3 \times 10^{10}$
\end{tabular}

\section{Preamp module}

High sensitivity of detection system was achieved by both matching the IR detector to the preamp and minimizing noises. Figure 4 shows the simulation diagram of the detection system.

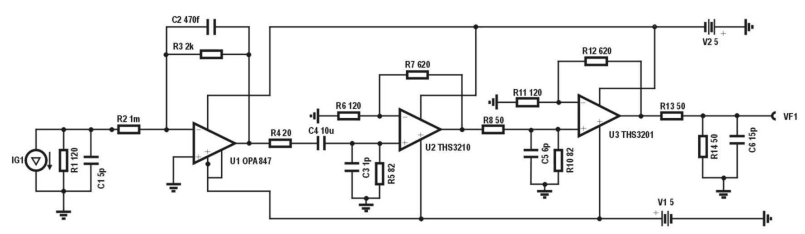

Fig. 4. Simulation diagram of the detection system.

The current signal from the photodiode is read by a broadband transimpedance preamp with resultant transimpedance of $27 \mathrm{kV} / \mathrm{A}$.

Preamp simulations have been carried out using the TINA TI software. The noise and signal characteristics are determined (Fig. 5). Based on these characteristics, it is possible to choose the operating point of the detection module for the highest signal to noise ratio while maintaining high signal gain. Signal gain for this system is amounted to $130 \mathrm{~Hz}-200 \mathrm{MHz}$.

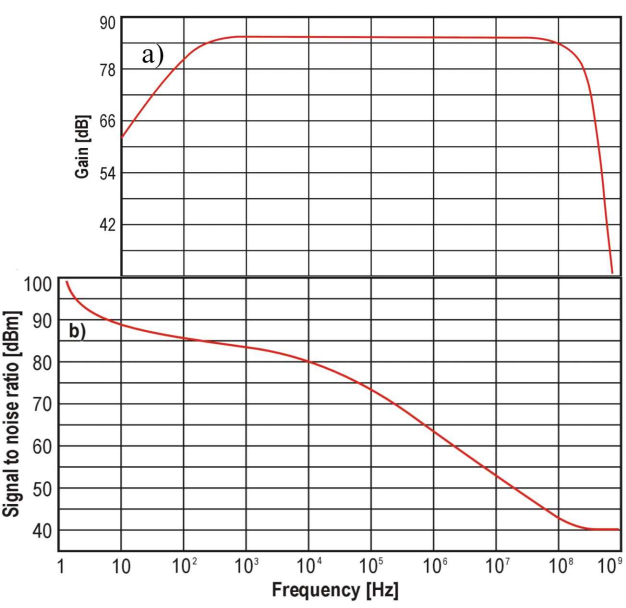

Fig. 5. Frequency characteristics of signal gain (a) and signal to noise ratio (b).

The preamp noises and signal characteristics have been tested using the spectrum analyzer GSP-830. Test results are shown in Figs. 6 and 7. In the noise characteristic the $1 / f$ noises range is noticed.

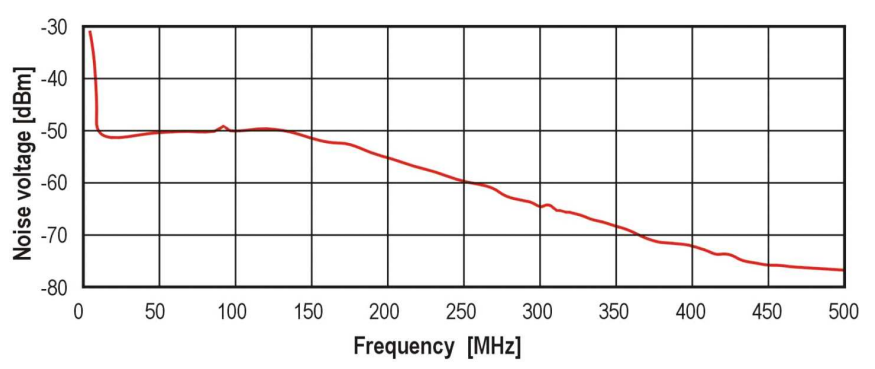

Fig. 6. Noise voltage characteristics of the preamp module.

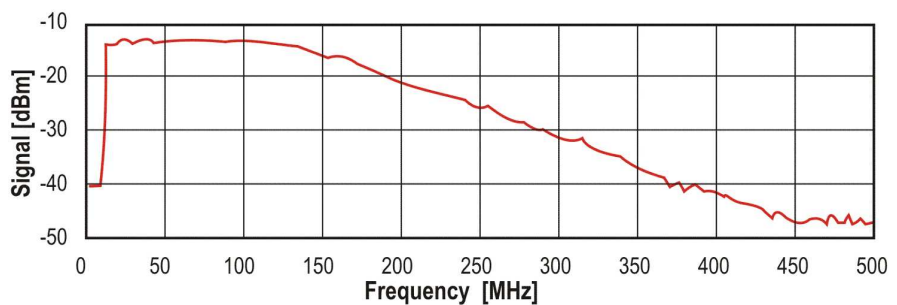

Fig. 7. Signal characteristics of the preamplifier module.

The signal characteristics show that the photodetectors $\mathrm{AC}$ bandwidth is of value of $150 \mathrm{MHz}$.

\section{Detection module}

The detection module consists of a radiation detector, four-stage thermoelectric cooler with temperature sensor, 


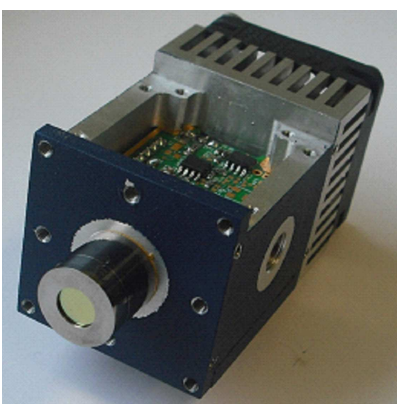

Fig. 8. View of the detection module.

broadband transimpedance preamp, and TEC controller (Fig. 8).

The detection module noises were tested on the spectrum analyzer GSP-830. Test results are shown in Fig. 9. The shape of the noise spectrum for the detection module is comparable to preamp one.

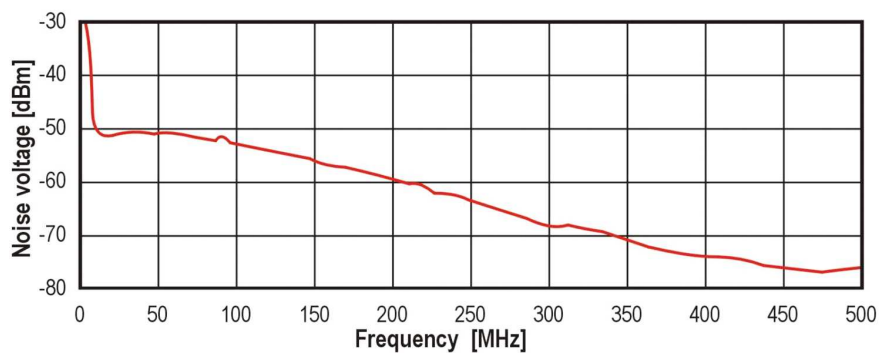

Fig. 9. Noise voltage characteristics of the detection module.

The time constant (signal frequency bandwidth) of the detection module were also measured. For this purpose, a QCL laser system LM-03-D produced by Cascade Technologies company was applied. The laboratory setup is presented in Fig. 10.

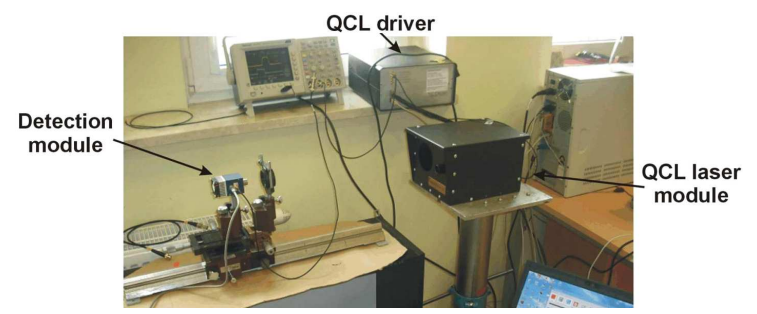

Fig. 10. Lab setup to measure the parameters of the laser pulses.

Based on the shape of recorded pulses from the detection module, frequency limit was determined (Fig. 11.)

The calculations took into account the measured value of the signal fall time of the module, time parameters of laser pulses and the parameters of the oscilloscope. The detection module detectivity was once more tested on the spectrophotometer. Test results are shown in Fig. 12.

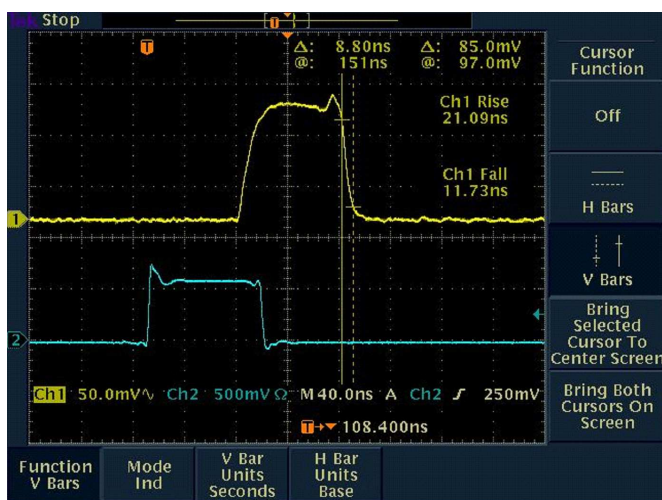

Fig. 11. Registered signals from the detection module (Ch 1) and laser trigger output (Ch 2).

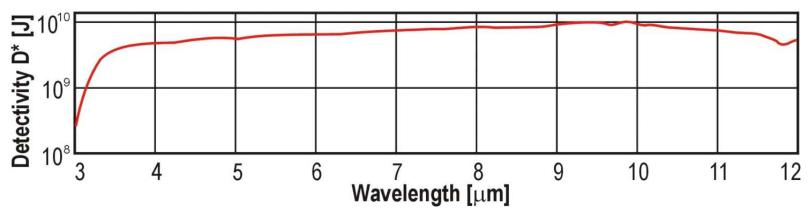

Fig. 12. Detectivity characteristics of the detection module.

\section{Summary}

The described detection module is designed for operation at the wavelength of $10 \mu \mathrm{m}$. The used detectors have been manufactured by Vigo System S.A. Co. High sensitivity is obtained by both, a multi-layer $\mathrm{Hg}_{1-x} \mathrm{Cd}_{x} \mathrm{Te}$ heterostructure, and an immersion lens. To decrease the noise level the detector was equipped with a four-stage thermoelectric cooler. It will provide to design a second generation FSO. This optical link gives opportunity to obtain better range in bad weather conditions as compared to the currently available ones.

\section{Acknowledgments}

The authors would like to thank the Ministry of Science and Higher Education for their support in the field. The article was developed under research grant No. O R00 00 8606.

\section{References}

[1] Z. Bielecki, W. Kołosowski, J. Mikołajczyk, M. Nowakowski, PIERS, Conf. Proc., Boston (USA) 2008, p. 95, www . emacademy . org .

[2] J. Mikołajczyk, Z. Bielecki, M. Nowakowski, J. Wojtas, Sensor+Test 2008 Proc., Nurnberg (Germany) 2008, p. 313.

[3] Z. Bielecki, W. Kołosowski, J. Mikołajczyk, M. Nowakowski, E. Sędek, J. Wojtas, in: XVIII Int. Conf. on Microwaves, Radar and Wireless Communications, Conf. Proc., Wrocław (Poland) 2008, Vol. 2, p. 295. 
[4] X. Liu, The Free-Space Optics System Using QCL: Models and Solutions, International Conference on Communications 2007, p. 2457.

[5] A. Piotrowski, P. Madejczyk, W. Gawron, K. Klos, M. Romanis, M. Grudzien, J. Piotrowski, A. Rogalski, Bul. Pol. Ac. Tech. 53, 139 (2005).

[6] P. Madejczyk, A. Piotrowski, W. Gawron, K. Kłos, J. Pawluczyk, J. Rutkowski, J. Piotrowski, A. Rogalski, Opt. Electron. Rev. 13, 239 (2005).

[7] A. Piotrowski, P. Madejczyk, W. Gawron, K. Kłos, J. Pawluczyk, J. Rutkowski, J. Piotrowski, A. Rogalski, Infrared Phys. Technol. 49, 173 (2007).

[8] W. Gawron, A. Piotrowski, K. Kłos, Z. Orman, J. Pawluczyk, D. Stanaszek, A. Kębłowski, M. Pędzińska, J. Piotrowski, Elektronika 5, 61 (2009).
[9] A. Piotrowski, J. Piotrowski, W. Gawron, J. Pawluczyk, M. Pedzinska, Acta Phys. Pol. A 116, 52 (2009).

[10] A. Rogalski, Acta Phys. Pol. A 116, 389 (2009).

[11] D. Stanaszek, J. Piotrowski, A. Piotrowski, W. Gawron, Z. Orman, R. Paliwoda, M. Brudnowski, J. Pawluczyk, M. Pedzińska, Proc. SPIE 7482, 74820M (2009); DOI: 10.1117/12.835963. 